\title{
Helicoidal Surfaces and Their Relationship to Bonnet Surfaces
}

\author{
Paul Bracken \\ Department of Mathematics, University of Texas, Edinburg, TX, USA \\ Email:paul.bracken@utrgv.edu
}

How to cite this paper: Bracken, P. (2017) Helicoidal Surfaces and Their Relationship to Bonnet Surfaces. Advances in Pure Mathematics, 7, 31-40.

http://dx.doi.org/10.4236/apm.2017.71003

Received: October 20, 2016

Accepted: January 21, 2017

Published: January 24, 2017

Copyright $\odot 2017$ by author and Scientific Research Publishing Inc. This work is licensed under the Creative Commons Attribution International License (CC BY 4.0).

http://creativecommons.org/licenses/by/4.0/

\begin{abstract}
An important question that arises is which surfaces in three-space admit a mean curvature preserving isometry which is not an isometry of the whole space. This leads to a class of surface known as a Bonnet surface in which the number of noncongruent immersions is two or infinity. The intention here is to present a proof of a theorem using an approach which is based on differential forms and moving frames and states that helicoidal surfaces necessarily fall into the class of Bonnet surfaces. Some other results are developed in the same manner.
\end{abstract}

\section{Keywords}

Surface, Fundamental Forms, Structure Equations, Mean Curvature, Bonnet, Helicoidal

\section{Introduction}

Surfaces that admit isometries which preserve principal curvatures have been studied since the time of O. Bonnet [1]. It was shown by Bonnet that all surfaces with constant mean curvature not including planes and spheres, can be isometrically deformed while preserving mean curvature or equivalently both principal curvatures. Let $\Sigma$ denote the classes of smooth oriented, connected surfaces carrying a Riemannian metric which will be studied here. Let $H: \Sigma \rightarrow \mathbb{R}$ be a given smooth function, then depending on the surface and the function $H$, it is possible to find isometric immersions of $\Sigma$ into $\mathbb{R}^{3}$ such that each image has mean curvature function $H$. It may be asked how many geometrically distinct, or noncongruent, immersions exists. This question has been studied at various levels both locally and globally. It has been concluded that the number of noncongruent immersions which is denoted here by $N$, may be $0,1,2$ and $\infty$. The case in which $N=\infty$ means there exists a 
one-parameter family of pairwise noncongruent isometric immersions with the same mean curvature function. When $N=2$ or $\infty$, these surfaces are the so-called Bonnet surfaces. When $N=0$ or 1 , there are many well-known surfaces, so all of these numbers can be realized.

Some of the global results that have been reported up to this point should be reviewed first. 1) If $H$ is constant and there is an isometric immersion of $\Sigma$ into $\mathbb{R}^{3}$ of mean curvature $H$ which is not a plane or sphere, then there is an isometric deformation of $\Sigma$ through noncongruent surfaces with the same constant mean curvature, $H$. 2) If $\Sigma$ is compact and $H$ is nonconstant, there exists at most two geometrically distinct immersions of $\Sigma$ in $\mathbb{R}^{3}$ with mean curvature $H$, so $N=0,1$ or 2 . If $\Sigma$ is homeomorphic to $S^{2}$, the 2-sphere, there is at most one isometric immersion, and $N$ is 0 or 1.3) If $\Sigma$ is a helicoidal surface in $\mathbb{R}^{3}$, then $N=2$ or $\infty$. 4) If the Gaussian curvature $K$ of $\Sigma$ is a nonzero constant, and the mean curvature $H$ is nonconstant, then $N=0,1$ or 2 .

The surfaces in Euclidean space that admit a mean curvature preserving isometry which is not an isometry of the whole space form a special class of surface which has been studied by many people such as noted already Bonnet as well as Cartan and Chern [2] [3] [4]. These surfaces may be broken up into three classes or types which can be described as follows: 1) There are surfaces of constant mean curvature other than the plane or sphere 2) There are certain surfaces of nonconstant mean curvature which admit a one-parameter family of geometrically distinct nontrivial isometries, and finally 3) There are surfaces of nonconstant mean curvature that admit a single nontrivial isometry which is unique up to an isometry of the entire space.

A surface that belongs to one of the above types is called a Bonnet surface, that is, an $N=2, \infty$ type mentioned above [5] [6] [7]. By a nontrivial isometry of the surface is meant an isometry of the entire space. A helicoidal surface in Euclidean three-space $E^{3}$ is the locus of an appropriately chosen curve under a helicoidal motion, with so-called pitch in the interval $(0, \infty)$ [8] [9]. Such a motion can be described by a one-parameter group of isometries in $E^{3}$. The actual orbits of the motion through the initial curve foliate the surface.

The intention here is to prove that the helicoidal surfaces are necessarily Bonnet surfaces, and moreover represent all three types of surface outlined above. Although not all the theorems presented here are new, the objective is to present new proofs based on the systematic use of differential forms and the moving frame concept [7]. This type of result is useful to have since it provides an answer, in the negative, to conjectures such as the following: Let $S$ be a Riemannian surface and $H: S \rightarrow \mathbb{R}$ a smooth function. If a nontrivial family of isometric immersions with mean curvature function $H$ does not exist, then there must be at most two noncongruent ones. Then it may be conjectured: In the absence of a nontrivial family, the immersion must be unique. On the other hand, it seems that not all Bonnet surfaces of the third type are helicoidal surfaces. A helicoidal surface is determined by one real-valued function of one 
variable, whereas a Bonnet surface of the third kind depends on four functions of one variable and therefore has a greater degree of generality [10].

\section{Structure Equations}

Over $\Sigma$ there exists a system of orthonormal frames $\left\{x, e_{1}, e_{2}, e_{3}\right\}$ which is well defined such that $x \in \Sigma, e_{3}$ is the unit normal at $x$ and $e_{1}, e_{2}$ located along principal directions. The fundamental equations for a surface have the form [11],

$\mathrm{d} x=\omega_{1} e_{1}+\omega_{2} e_{2}, \quad \mathrm{~d} e_{1}=\omega_{12} e_{2}+\omega_{13} e_{3}, \quad \mathrm{~d} e_{2}=-\omega_{12} e_{1}+\omega_{23} e_{3}, \quad \mathrm{~d} e_{3}=-\omega_{13} e_{1}-\omega_{23} e_{2}$.

These equations can be differentiated exteriorly in turn and results in a large system of equations for the exterior derivatives of the $\omega_{i}$ and $\omega_{i j}$, as well as a final equation which relates some of the forms. This choice of frame and Cartan's lemma allows for the introduction of the two principal curvatures at $x$ which are denoted by $a$ and $c$ by writing

$$
\omega_{12}=h \omega_{1}+k \omega_{2}, \quad \omega_{13}=a \omega_{1}, \quad \omega_{23}=c \omega_{2} .
$$

It suffices to suppose that $a>c$ in the following and the mean curvature of $\Sigma$ will be denoted by $H$ and the Gaussian curvature is denoted by $K$. They are defined in terms of the functions $a$ and $c$ to be

$$
H=\frac{1}{2}(a+c), \quad K=a \cdot c .
$$

The forms which appear in (2.1) satisfy the fundamental set of structure equations

$$
\begin{aligned}
& \mathrm{d} \omega_{1}=\omega_{12} \wedge \omega_{2}, \quad \mathrm{~d} \omega_{2}=\omega_{1} \wedge \omega_{12}, \\
& \mathrm{~d} \omega_{13}=\omega_{12} \wedge \omega_{23}, \quad \mathrm{~d} \omega_{23}=\omega_{13} \wedge \omega_{12}, \\
& \mathrm{~d} \omega_{12}=a c \omega_{2} \wedge \omega_{1}=-K \omega_{1} \wedge \omega_{2} .
\end{aligned}
$$

The second pair of equations in (2.4) is referred to as the Codazzi equation and the last equation is called the Gauss equation.

Exterior differentiation of the Codazzi equations in (2.4) and using (2.2) yields

$$
\left(\mathrm{d} a-(a-c) h \omega_{2}\right) \wedge \omega_{1}=0, \quad\left(\mathrm{~d} c-(a-c) k \omega_{1}\right) \wedge \omega_{2}=0 .
$$

Now Cartan's lemma can be applied to (2.5). There exist two functions $u$ and $v$ such that

$$
\frac{1}{a-c} \mathrm{~d} a-h \omega_{2}=(u-k) \omega_{1}, \quad \frac{1}{a-c} \mathrm{~d} c-k \omega_{1}=(v-h) \omega_{2} .
$$

Subtracting the pair of equations in (2.6) gives an expression for $\mathrm{d} \log (a-c)$,

$$
\mathrm{d} \log (a-c)=(u-2 k) \omega_{1}-(v-2 h) \omega_{2} \text {. }
$$

It is natural from (2.7) to define a new variable $J$ in terms of $a$ and $a c$ as

$$
J=\frac{1}{2}(a-c)>0 .
$$

Equation (2.7) can then be put in the form, 


$$
\operatorname{dlog} J=(u-2 k) \omega_{1}-(v-2 h) \omega_{2} .
$$

The differential forms $\omega_{i}$ constitute a linearly independent system. Two related coframes $\vartheta_{i}$ and $\alpha_{i}$ can be defined in terms of the $\omega_{i}$ and the functions $u$ and $v$ as follows

$$
\begin{array}{ll}
\vartheta_{1}=u \omega_{1}+v \omega_{2}, & \vartheta_{2}=-v \omega_{1}+u \omega_{2}, \\
\alpha_{1}=u \omega_{1}-v \omega_{2}, & \alpha_{2}=v \omega_{1}+u \omega_{2} .
\end{array}
$$

These relations imply that $\vartheta_{1}=0$ is tangent to the level curves which are singled out by setting $H$ equal to a constant and $\alpha_{1}=0$ is its symmetry with respect to the original directions.

The relation $2 H=a+c$ is squared and subtracting the definition of the Gaussian curvature, $4 K=4 a c$ yields the result $4\left(H^{2}-K\right)=(a-c)^{2}$. The Hodge operator, denoted here by $*$, will play an important role in the following. It produces the following result on the basis forms $\omega_{i}$ in (2.2),

$$
* \omega_{1}=\omega_{2}, \quad * \omega_{2}=-\omega_{1}, \quad *^{2}=-1 .
$$

From these properties, the dual relations can be determined as

$$
\begin{array}{ll}
* \vartheta_{1}=u \omega_{2}-v \omega_{1}=\vartheta_{2}, & * \vartheta_{2}=-v \omega_{2}-u \omega_{1}=-\vartheta_{1}, \\
* \alpha_{1}=u \omega_{2}+v \omega_{1}=\alpha_{2}, & * \alpha_{2}=v \omega_{2}-u \omega_{1}=-\alpha_{1} .
\end{array}
$$

Moreover, adding the expressions for $\mathrm{d} a$ and $\mathrm{d} c$ given by (2.6), we obtain

$$
\frac{1}{a-c}(\mathrm{~d} a+\mathrm{d} c)=(u-k) \omega_{1}+h \omega_{2}+(v-h) \omega_{2}+k \omega_{1}=u \omega_{1}+v \omega_{2}=\vartheta_{1}
$$

Finally, there is the relation,

$\alpha_{1}+2 * \omega_{12}=u \omega_{1}-v \omega_{2}+2 *\left(h \omega_{1}+k \omega_{2}\right)=(u-2 k) \omega_{1}-(v-2 h) \omega_{2}=d \log J$.

Therefore, the Codazzi Equations (2.12) and (2.13) can be summarized in terms of the two functions $H$ and $J$ as follows,

$$
d H=J \vartheta_{1}, \quad d \log J=\alpha_{1}+2 * \omega_{12} .
$$

\section{Bonnet Surfaces}

Suppose that $\Sigma^{*}$ is a surface which is isometric to $\Sigma$ such that the principal curvatures are preserved under the transformation. Denote all quantities which pertain to $\Sigma^{*}$ by the same symbols, but with an asterisk,

$$
a^{*}=a, \quad c^{*}=c .
$$

The same convention will be applied to the variables and forms which pertain to $\Sigma$ and $\Sigma^{*}$. When $\Sigma$ and $\Sigma^{*}$ are isometric, the forms $\omega_{i}$ on $\Sigma$ are related to the forms $\omega_{i}^{*}$ on $\Sigma^{*}$ by means of the transformation

$$
\omega_{1}^{*}=\cos \tau \omega_{1}-\sin \tau \omega_{2}, \quad \omega_{2}^{*}=\sin \tau \omega_{1}+\cos \tau \omega_{2} .
$$

The following theorem from [7] will be required.

Theorem 3.1: Under the transformation of coframe given in (3.2), the associated connection forms are related by

$$
\omega_{12}^{*}=\omega_{12}-\mathrm{d} \tau \text {. }
$$


There is a very important result which can be developed at this point. In the case that $a=a^{*}$ and $c=c^{*}$, the Codazzi equations imply that

$$
\alpha_{1}+2 * \omega_{12}=\operatorname{dlog}(a-c)=\operatorname{dlog}\left(a^{*}-c^{*}\right)=\alpha_{1}^{*}+2 * \omega_{12}^{*} .
$$

Now apply the operator $*$ to both sides of this equation to give,

$$
\alpha_{2}-2 \omega_{12}=\alpha_{2}^{*}-2 \omega_{12}^{*} \text {. }
$$

Substituting for $\omega_{12}^{*}$ from Theorem 3.1, this assumes the form,

$$
2 \mathrm{~d} \tau=\alpha_{2}-\alpha_{2}^{*} .
$$

Define $H_{1}$ and $H_{2}$ to be the derivatives of the function $H$ in directions such that $\mathrm{d} H$ can be expanded as

$$
\mathrm{d} H=H_{1} \omega_{1}+H_{2} \omega_{2} .
$$

Since $d H=J \vartheta_{1}$ and using $\vartheta_{1}$ given by (2.10), Equation (2.13) produces

$$
u \omega_{1}+v \omega_{2}=\frac{H_{1}}{J} \omega_{1}+\frac{H_{2}}{J} \omega_{2},
$$

Comparing coefficients of $\omega_{1}$ and $\omega_{2}$ on both sides, we can identify

$$
u=\frac{H_{1}}{J}, \quad v=\frac{H_{2}}{J} .
$$

This result implies that

$$
\alpha_{1}=\frac{H_{1}}{J} \omega_{1}-\frac{H_{2}}{J} \omega_{2}, \quad \alpha_{2}=\frac{H_{2}}{J} \omega_{1}+\frac{H_{1}}{J} \omega_{2} .
$$

Since $\alpha_{2}^{*}=\sin (2 \tau) \alpha_{1}+\cos (2 \tau) \alpha_{2}$, it follows from (3.4) that

$$
\alpha_{2}-\mathrm{d}(2 \tau)=\sin (2 \tau) \alpha_{1}+\cos (2 \tau) \alpha_{2} .
$$

Solving this for $\mathrm{d}(2 \tau)$ and substituting for $\alpha_{1}$ and $\alpha_{2}$, it follows that

$$
\begin{aligned}
\mathrm{d}(2 \tau) & =(1-\cos (2 \tau)) \alpha_{2}-\sin (2 \tau) \alpha_{1} \\
& =-\sin (2 \tau)\left(\frac{H_{1}}{J} \omega_{1}-\frac{H_{2}}{J} \omega_{2}\right)-\cos (2 \tau)\left(\frac{H_{2}}{J} \omega_{1}+\frac{H_{1}}{J} \omega_{2}\right)+\alpha_{2} \\
& =-\sin (2 \tau)\left(\frac{H_{1}}{J} \omega_{1}-\frac{H_{2}}{J} \omega_{2}\right)-\cos (2 \tau)\left(\frac{H_{2}}{J} \omega_{1}+\frac{H_{1}}{J} \omega_{2}\right)+{ }^{*} \alpha_{1} .
\end{aligned}
$$

Therefore, using the second equation of (2.14) for $\alpha_{1}$ implies that

$$
\mathrm{d}(2 \tau)=-\sin (2 \tau)\left(\frac{H_{1}}{J} \omega_{1}-\frac{H_{2}}{J} \omega_{2}\right)-\cos (2 \tau)\left(\frac{H_{2}}{J} \omega_{1}+\frac{H_{1}}{J} \omega_{2}\right)+* \operatorname{dlog} J+2 \omega_{12} \text {. }
$$

The differential in (3.6) will play a role in the study of helicoidal surfaces.

\section{Helicoidal Surfaces}

Every helicoidal surface can be parametrized in terms of two parameters $(s, t)$, where $t$ can be thought of as time along orbits from a fixed $t=t_{0}$, and $s$ is an arc-length of curves orthogonal to orbits. Then the curves $t=c$ are carried along the orbits by the helicoidal motion for $c$ constant. They remain orthogonal to the orbits and foliate the surface. An orthonormal frame $e_{1}, e_{2}$ 
is determined along these coordinate curves. The corresponding coframe may be written as

$$
\omega_{1}=\mathrm{d} s, \quad \omega_{2}=q(s) \mathrm{d} t,
$$

where $q$ depends only on $s$. Since $\mathrm{d} \omega_{1}=0$, the equation $\mathrm{d} \omega_{1}=\omega_{12} \wedge \omega_{2}$ implies that $\omega_{12}$ is proportional to $\mathrm{d} t$, say $f \mathrm{~d} t$ and $\mathrm{d} \omega_{2}=\mathrm{d} s \wedge \omega_{12}=f \mathrm{~d} s \wedge \mathrm{d} t$ implies that

$$
q^{\prime}(s) \mathrm{d} s \wedge \mathrm{d} t=f \mathrm{~d} s \wedge \mathrm{d} t,
$$

so,

$$
\omega_{12}=q^{\prime}(s) \mathrm{d} t=\frac{q^{\prime}(s)}{q(s)} \omega_{2} .
$$

Hence, the $\omega_{1}$-curves are geodesics, and the $\omega_{2}$-curves or orbits, have geodesic curvature equal to

$$
\mu(s)=\frac{\mathrm{d}}{\mathrm{d} s} \log (|q(s)|) .
$$

Thus along each orbit, the quantities $a, c, \mu$ and $\tau$ are constant and depend only on $s$. In this case, the derivative $H_{2}=0$. Also for the same reason, the differential form $\mathrm{d} J=J_{1} \omega_{1}+J_{2} \omega_{2}$ of $J$ implies that $J_{2}=0$, hence

$$
\operatorname{dlog} J=\left(\frac{J_{1}}{J}\right) \omega_{1} .
$$

Hence, the equation for $\mathrm{d}(2 \tau)$ in (3.6) takes the form

$$
\mathrm{d}(2 \tau)=-\sin (2 \tau) \frac{H_{1}}{J} \omega_{1}-\cos (2 \tau) \frac{H_{1}}{J} \omega_{2}+* \frac{J_{1}}{J} \omega_{1}+2 \mu(s) \omega_{2},
$$

that is,

$$
\mathrm{d}(2 \tau)=-\sin (2 \tau) \frac{H_{1}}{J} \omega_{1}-\cos (2 \tau) \frac{H_{1}}{J} \omega_{2}+\frac{J_{1}}{J} \omega_{2}+2 \mu(s) \omega_{2} .
$$

Writing $\mathrm{d}(2 \tau)$ as a differential form in terms of $\mathrm{d} s$ and $\mathrm{d} t$ and then equating coefficients of $\mathrm{d} s$ and $\mathrm{d} t$ on both sides of (4.5) yields the following pair of equations,

$$
\frac{\mathrm{d}(2 \tau)}{\mathrm{d} s}=-\sin (2 \tau) J^{-1} \frac{\mathrm{d} H}{\mathrm{~d} s}, \quad 2 \mu(s)=\cos (2 \tau) J^{-1} H_{1}-J^{-1} J_{1} .
$$

The results in (4.6) are used in the proof of Theorem 5.2 which follows.

\section{Main Theorems and Proofs}

Now by what has been established so far, both functions $a$ and $c$ depend on the variable $s$, so this mapping is an isometry which preserves $H$, since $H$ is the average of $a$ and $c$. In general, an isometry is trivial if and only if it preserves the mean curvature and the principal directions. In this case, the above mapping is trivial if and only if $\tau$ is a multiple of $\pi / 2$. Then we obtain that the orbits are plane curves. But this is impossible for a helicoidal surface. This proves the following result. 
Theorem 5.1. For a helicoidal surface, the mapping $(s, t) \rightarrow(s,-t)$ is a nontrivial isometry which preserves the mean curvature $H$.

To prove the second theorem, the following result due to Chern is required [4] [7].

Proposition 5.1. (Chern) A surface $M$ admits a nontrivial isometric deformation that keeps the principal curvatures fixed if and only if

$$
\mathrm{d} \alpha_{1}=0, \quad \mathrm{~d} \alpha_{2}=\alpha_{1} \wedge \alpha_{2} .
$$

Theorem 5.2. A helicoidal surface is a Bonnet surface of the second type if and only if the following relation is satisfied,

$$
\frac{\mathrm{d}}{\mathrm{d} s}\left(\frac{1}{J} \frac{\mathrm{d} H}{\mathrm{~d} s}\right)-\left(\frac{1}{J} \frac{\mathrm{d} H}{\mathrm{~d} s}\right)^{2}(\cos \varphi(s))+\frac{1}{J} \frac{\mathrm{d} H}{\mathrm{~d} s} \frac{\mathrm{d} \log |q(s)|}{\mathrm{d} s}=0 .
$$

with $H=H(s)$ nonconstant.

Proof: Set $\varphi=2 \tau$ and consider the principal coframe

$$
\omega_{1}=\cos \varphi(s) \mathrm{d} s+\sin \varphi(s) q(s) \mathrm{d} t, \quad \omega_{2}=-\sin \varphi(s) \mathrm{d} s+\cos \varphi(s) q(s) \mathrm{d} t .
$$

Define $H_{1}$ and $H_{2}$ as the coefficients in the differential $\mathrm{d} H$ by putting $\mathrm{d} H=H_{1} \omega_{1}+H_{2} \omega_{2}$ and let $\alpha_{1}$ and $\alpha_{2}$ be given by (3.5). Next we substitute $\alpha_{1}$ and $\alpha_{2}$ into the equations which appear in Chern's result given in Proposition 5.1. Since $H_{2}=0$ in the $(\mathrm{d} s, \mathrm{~d} t)$ basis of forms, it follows that

$$
\begin{aligned}
\mathrm{d} \alpha_{1} & =\mathrm{d}\left(\frac{H_{1}}{J}\right) \wedge \omega_{1}+\frac{H_{1}}{J} \mathrm{~d} \omega_{1}=\frac{\mathrm{d}}{\mathrm{d} s}\left(\frac{H_{1}}{J}\right) \mathrm{d} s \wedge \omega_{1}+\frac{H_{1}}{J} \frac{\mathrm{d}}{\mathrm{d} s}(\sin \varphi(s) q(s)) \mathrm{d} s \wedge \mathrm{d} t \\
& =\frac{\mathrm{d}}{\mathrm{d} s}\left(\frac{H_{1}}{J}\right) \sin \varphi q(s) \mathrm{d} s \wedge \mathrm{d} t+\frac{H_{1}}{J}\left(\cos \varphi \frac{\mathrm{d} \varphi}{\mathrm{d} s} q+\sin \varphi \frac{\mathrm{d} q}{\mathrm{~d} s}\right) \mathrm{d} s \wedge \mathrm{d} t .
\end{aligned}
$$

Since the first equation is $\mathrm{d} \alpha_{1}=0$, this implies that

$$
\frac{H_{1}}{J}\left(\cos \varphi(s) \frac{\mathrm{d} \varphi}{\mathrm{d} s}\right)=-\frac{H_{1}}{J} \sin \varphi(s) \frac{\mathrm{d}}{\mathrm{d} s} \log |q(s)|-\frac{\mathrm{d}}{\mathrm{d} s}\left(\frac{H_{1}}{J}\right) \sin \varphi .
$$

Similarly, using (3.5), we have

$$
\begin{aligned}
& \mathrm{d} \alpha_{2}=\frac{\mathrm{d}}{\mathrm{d} s}\left(\frac{H_{1}}{J}\right) \mathrm{d} s \wedge \omega_{2}+\frac{H_{1}}{J} \mathrm{~d} \omega_{2} \\
&=\frac{\mathrm{d}}{\mathrm{d} s}\left(\frac{H_{1}}{J}\right) \cos \varphi(s) q(s) \mathrm{d} s \wedge \mathrm{d} t+\frac{H_{1}}{J} \frac{\mathrm{d}}{\mathrm{d} s}(\cos \varphi(s) q(s)) \mathrm{d} s \wedge \mathrm{d} t, \\
& \alpha_{1} \wedge \alpha_{2}=\left(\frac{H_{1}}{J}\right)^{2} q(s) \mathrm{d} s \wedge \mathrm{d} t .
\end{aligned}
$$

Equating these two results as in the second of Chern's two equations, we obtain

$$
\frac{\mathrm{d}}{\mathrm{d} s}\left(\frac{H_{1}}{J}\right) \cos \varphi(s)-\frac{H_{1}}{J} \sin \varphi(s) \frac{\mathrm{d} \varphi}{\mathrm{d} s}+\frac{H_{1}}{J} \cos \varphi(s) \frac{\mathrm{d}}{\mathrm{d} s} \log |q(s)|=\left(\frac{H_{1}}{J}\right)^{2} .
$$

Multiplying (5.3) by $\sin \varphi$ and (5.4) by $\cos \varphi$, it is found that the folowing hold:

$$
\frac{H_{1}}{J}\left(\sin \varphi \cos \varphi \frac{\mathrm{d} \varphi}{\mathrm{d} s}\right)=-\frac{H_{1}}{J} \sin ^{2} \varphi \frac{\mathrm{d}}{\mathrm{d} s} \log |q(s)|-\frac{\mathrm{d}}{\mathrm{d} s}\left(\frac{H_{1}}{J}\right) \sin ^{2} \varphi,
$$




$$
\cos ^{2} \frac{\mathrm{d}}{\mathrm{d} s}\left(\frac{H_{1}}{J}\right)-\frac{H_{1}}{J}\left(\cos \varphi \sin \varphi \frac{\mathrm{d} \varphi}{\mathrm{d} s}\right)+\frac{H_{1}}{J} \cos ^{2} \varphi \frac{\mathrm{d}}{\mathrm{d} s} \log |q(s)|=\left(\frac{H_{1}}{J}\right)^{2} \cos \varphi .
$$

Adding these two equations, the desired result is obtained,

$$
\frac{\mathrm{d}}{\mathrm{d} s}\left(\frac{H_{1}}{J}\right)-\left(\frac{H_{1}}{J}\right)^{2} \cos \varphi(s)+\frac{H_{1}}{J} \frac{\mathrm{d}}{\mathrm{d} s} \log |q(s)|=0 .
$$

Replacing $H_{1}$ by $\mathrm{d} H / \mathrm{d} s$ in (5.5), equation (5.1) follows.

Multiply (5.5) by $q(s) \sin \varphi(s)$ to obtain,

$$
J \frac{\mathrm{d}}{\mathrm{d} s}\left(J^{-1} \frac{\mathrm{d} H}{\mathrm{~d} s}\right) \sin \varphi q(s)-\frac{\mathrm{d} H}{\mathrm{~d} s} \cos \varphi \sin \varphi \frac{\mathrm{d} H}{\mathrm{~d} s} J^{-1} q+\frac{\mathrm{d} H}{\mathrm{~d} s} \sin \varphi \frac{\mathrm{d} q}{\mathrm{~d} s}=0 .
$$

Substituting the derivative for $2 \tau$ into (5.6), it becomes,

$$
J \frac{\mathrm{d}}{\mathrm{d} s}\left(J^{-1} \frac{\mathrm{d} H}{\mathrm{~d} s}\right) \sin \varphi q(s)+\frac{\mathrm{d} H}{\mathrm{~d} s} \cos \varphi \frac{\mathrm{d} \varphi}{\mathrm{d} s} q(s)+\frac{\mathrm{d} H}{\mathrm{~d} s} \sin \varphi \frac{\mathrm{d} q}{\mathrm{~d} s}=0 .
$$

By means of the product rule, this can be put in the form,

$$
\frac{\mathrm{d}}{\mathrm{d} s}\left[\frac{1}{J} \frac{\mathrm{d} H}{\mathrm{~d} s} \sin \varphi(s) q(s)\right]=0 \text {. }
$$

This is trivial to integrate, so if $C$ is the integration constant, we obtain that

$$
\frac{1}{J} \frac{\mathrm{d} H}{\mathrm{~d} s} \sin \varphi(s) \cdot q(s)=C,
$$

with $H=H(s)$ nonconstant.

Since this relation may be viewed as an ordinary differential equation for the real-valued function which determines the helicoidal surface under helicoidal motion, the existence of such a surface is guaranteed by the local existence uniqueness theorem for solutions of such an ordinary differential equation.

From the first equation of (4.6) and the fact that the space curvature of orbits is either $\left[\mu^{2}+\left(a\left(\cos ^{2} \sigma\right)+c\left(\sin ^{2} \sigma\right)\right)^{2}\right]^{1 / 2}$ or this with $\sin -\cos$ interchanged, the last result follows.

Theorem 5.3. A helicoidal surface has constant mean curvature if and only if its principal directions make an angle constant with the orbits.

Combining all of these results, the main result of this work can be stated in the form of the following Theorem.

Theorem 5.4. The helicoidal surfaces are necessarily Bonnet surfaces and they represent all three types of surface.

A conclusion that follows from these results then is an interesting new geometric characterization of such surfaces. Thus, a helicoidal surface has constant mean curvature if and only if its principal directions make an angle which is constant with the orbits.

Finally, it will be proved that for any surface of revolution in $\mathbb{R}^{3}$ which has nonconstant mean curvature function $H$ it holds that either $N=1$ or $N=\infty$.

Let $x=r(z)>0$ be a plane curve in the $x-z$ plane and form the surface of revolution 


$$
r(z, \vartheta)=(r(z) \cos \vartheta, r(z) \sin \vartheta, z), \quad-\infty<\vartheta<\infty .
$$

The principal curvatures are calculated to be

$$
a=-\frac{r_{z z}}{\left[r_{z}^{2}+1\right]^{3 / 2}}, \quad c=\frac{1}{r\left[r_{z}^{2}+1\right]^{3 / 2}} .
$$

If $a=c$ at $p \in r(z, \vartheta)$, of course the entire parallel through $p$ consists of umbilic points, so $N=1$. Here $r_{z}$ denotes the derivative of $r(z)$ with respect to $Z$.

Theorem 5.5: Surfaces of revolution with nonconstant mean curvature that admit a one-parameter family of geometrically distinct nontrivial isometries preserving principal curvatures $a, c$ are exactly those for which the function $x=r(z)$ satisfies a specific fourth order differential equation in $z$.

Proof: For the surface of revolution of the form (5.9), the principal coframe is given by

$$
\omega_{1}=\sqrt{r_{z}^{2}+1}, \quad \omega_{2}=r(z) \mathrm{d} \vartheta
$$

Since principal curvatures $a$ and $c$ in (5.10) depend only on $Z$ and not on $\vartheta$, the first equation of (2.14) implies that

$$
(a+c)_{z} \mathrm{~d} z=(a-c)\left(u \sqrt{r_{z}^{2}+1} \mathrm{~d} z-v r(z) \mathrm{d} \vartheta\right) .
$$

Equating the coefficients of the differentials $\mathrm{d} z$ and $\mathrm{d} \vartheta$ on both sides gives $u$ and $v$,

$$
u=\frac{(a+c)_{z}}{(a-c) \sqrt{r_{z}^{2}+1}}, \quad v=0
$$

Then the forms $\alpha_{1}$ and $\alpha_{2}$ can be calculated from (2.10),

$$
\alpha_{1}=\frac{(a+c)_{z}}{(a-c)} \mathrm{d} z, \quad \alpha_{2}=\frac{(a+c)_{z} r(z)}{(a-c) \sqrt{r_{z}^{2}+1}} \mathrm{~d} \vartheta
$$

Substituting (5.14) into the differential expressions of Proposition 5.1, it is clear that $\mathrm{d} \alpha_{1}=0$ must always hold since the coefficient of $\alpha_{1}$ depends only on $\mathrm{z}$ and $\mathrm{dz} \wedge \mathrm{dz}=0$. To develope the second equation of the pair, we calculate $\mathrm{d} \alpha_{2}=\left[\frac{(a+c)_{z}}{(a-c) \sqrt{r_{z}^{2}+1}} r(z)\right]_{z} \mathrm{~d} z \wedge \mathrm{d} \vartheta, \quad \alpha_{1} \wedge \alpha_{2}=\frac{\left[(a+c)_{z}\right]^{2}}{(a-c)^{2}} \frac{r(z)}{\sqrt{r_{z}^{2}+1}} \mathrm{~d} z \wedge \mathrm{d} \vartheta$

Equating these two expressions, the following fourth-order differential equation for $r(z)$ is obtained,

$$
\left[\frac{(a+c)_{z}}{(a-c) \sqrt{r_{z}^{2}+1}} r(z)\right]_{z}=\frac{\left[(a+c)_{z}\right]^{2}}{(a-c)^{2}} \frac{r(z)}{\sqrt{r_{z}^{2}+1}} .
$$

In (5.16), the principal curvatures $a$ and $c$ are given in (5.10), and since $a$ contains second derivatives of $r$ with respect to $z$, equation (5.16) will be a fourth order equation in $z$. This is the equation mentioned in the Theorem. 


\section{References}

[1] Bonnet, O. (1867) Mémoire sur la theorie des surfaces applicables sur une surface donnée. J. l'École Pol., Paris, XLII Cahier, 72-92.

[2] Cartan, É. (1942) Sur les couples de surface applicables avec conservation des courbures principales. Bull. Sc. Math., 66, 55-85.

[3] Chern, S.S. (1955) An Elementary Proof of the Existence of Isothermal Parameters on a Surface. Proceedings of the American Mathematical Society, 6, 771-782. https://doi.org/10.1090/s0002-9939-1955-0074856-1

[4] Chern, S.S. (1985) Deformation of Surfaces Preserving Principal Curvatures. In: Rauch, H.E., Ed., Differential Geometry and Complex Analysis, Springer, Berlin, 155-163. https://doi.org/10.1007/978-3-642-69828-6_10

[5] Roussos, I.M. (1999) Global Results on Bonnet Surfaces. Journal of Geometry, 65, 151-168. https://doi.org/10.1007/BF01228686

[6] Bracken, P. (2008) Cartan's Theory of Moving Frames and an Application to a Theorem of Bonnet. Tensor, 70, 261-274.

[7] Bracken, P. (2017) An Intrinsic Characterization of Bonnet Surfaces Based on a Closed Differential Ideal. Manifolds-Current Research Areas, ed. P. Bracken, Intech Open Science Press, 21-43. https://doi.org/10.1155/2014/715679

[8] Roussos, I.M. (1988) The Helicoidal Surfaces as Bonnet Surfaces. Tohoku Mathematical Journal, Second Series, 40, 485-490.

[9] Roussos, I.M. (1987) Principal Curvature Preserving Isometries of Surfaces in Ordinary Space. Boletim da Sociedade Brasileira de Matemática, 18, 95-105. https://doi.org/10.1007/BF02590026

[10] Bobenko, A. and Eitner, U. (1998) Bonnet Surfaces and Painlevé Equations. Journal für die reine und angewandte Mathematik, 499, 47-79. https://doi.org/10.1515/crll.1998.061

[11] Chern, S.S., Chen, W.H. and Lam, K.S. (1999) Lectures in Differential Geometry, Vol. 1 of Series on University Mathematics. World Scientific, Singapore.

Submit or recommend next manuscript to SCIRP and we will provide best service for you:

Accepting pre-submission inquiries through Email, Facebook, LinkedIn, Twitter, etc. A wide selection of journals (inclusive of 9 subjects, more than 200 journals)

Providing 24-hour high-quality service

User-friendly online submission system

Fair and swift peer-review system

Efficient typesetting and proofreading procedure

Display of the result of downloads and visits, as well as the number of cited articles

Maximum dissemination of your research work

Submit your manuscript at: http://papersubmission.scirp.org/

Or contact apm@scirp.org 